\title{
Suzuki-Type Fixed Point Results in Metric-Like Spaces
}

\author{
Nabiollah Shobkolaei, ${ }^{1}$ Shaban Sedghi, ${ }^{2}$ Jamal Rezaei Roshan, ${ }^{2}$ and Nawab Hussain ${ }^{3}$ \\ ${ }^{1}$ Department of Mathematics, Babol Branch, Islamic Azad University, Babol, Iran \\ ${ }^{2}$ Department of Mathematics, Qaemshahr Branch, Islamic Azad University, Qaemshahr, Iran \\ ${ }^{3}$ Department of Mathematics, King Abdulaziz University, P.O. Box 80203, Jeddah 21589, Saudi Arabia
}

Correspondence should be addressed to Nawab Hussain; nhusain@kau.edu.sa

Received 16 May 2013; Accepted 17 July 2013

Academic Editor: Pei De Liu

Copyright (C) 2013 Nabiollah Shobkolaei et al. This is an open access article distributed under the Creative Commons Attribution License, which permits unrestricted use, distribution, and reproduction in any medium, provided the original work is properly cited.

We demonstrate a fundamental lemma for the convergence of sequences in metric-like spaces, and by using it we prove some Suzuki-type fixed point results in the setup of metric-like spaces. As an immediate consequence of our results we obtain certain recent results in partial metric spaces as corollaries. Finally, three examples are presented to verify the effectiveness and applicability of our main results.

\section{Introduction}

There are a lot of generalizations of Banach fixed-point principle in the literature. So far several authors have studied the problem of existence and uniqueness of a fixed point for mappings satisfying different contractive conditions (e.g., [120]). In 2008, Suzuki introduced an interesting generalization of Banach fixed-point principle. This interesting fixed-point result is as follows.

Theorem 1 (see [19]). Let $(X, d)$ be a complete metric space, and let $T$ be a mapping on $X$. Define a nonincreasing function $\theta$ from $[0,1]$ into $[1 / 2,1]$ by

$$
\theta(r)= \begin{cases}1, & 0 \leq r \leq \frac{\sqrt{5}-1}{2} \\ \frac{1-r}{r^{2}}, & \frac{\sqrt{5}-1}{2} \leq r \leq \frac{1}{\sqrt{2}} \\ \frac{1}{1+r}, & \frac{1}{\sqrt{2}} \leq r<1 .\end{cases}
$$

Assume that there exists $r \in[0,1]$, such that

$$
\theta(r) d(x, T x) \leq d(x, y) \Longrightarrow d(T x, T y) \leq r d(x, y),
$$

for all $x, y \in X$, then there exists a unique fixed-point $z$ of $T$. Moreover, $\lim _{n \rightarrow \infty} T^{n} x=z$ for all $x \in X$.
Suzuki proved also the following version of Edelstein's fixed point theorem.

Theorem 2. Let $(X, d)$ be a compact metric space. Let $T: X \rightarrow$ $X$ be a self-map, satisfying for all $x, y \in X, x \neq y$ the condition

$$
\frac{1}{2} d(x, T x) \leq d(x, y) \Longrightarrow d(T x, T y)<d(x, y) \text {. }
$$

Then $T$ has a unique fixed point in $X$.

This theorem was generalized in [3].

In addition to the above results, Kikkawa and Suzuki [8] provided a Kannan type version of the theorems mentioned before. In [14], Chatterjea type version is provided. Popescu [15] presented a Cirić type version. Recently, Kikkawa and Suzuki also provided multivalued versions which can be found in $[9,10]$.

Very recently Hussain et al. [4] have extended Suzuki's Theorems 1 and 2, as well as Popescu's results from [15] to the case of metric type spaces and cone metric type spaces (see also $[5-7,11])$.

The aim of this paper is to generalize the above-mentioned results. Indeed we prove a fixed point theorem in the set up of metric-like spaces and derive certain new results as corollaries. Finally, three examples are presented to verify the effectiveness and applicability of our main results. 
In the rest of this section, we recall some definitions and facts which will be used throughout the paper. First, we present some known definitions and propositions in partial metric and metric-like spaces.

A partial metric on a nonempty set $X$ is a mapping $p$ : $X \times X \rightarrow \mathbb{R}^{+}$such that for all $x, y, z \in X$,

$$
\begin{aligned}
& \left(\mathrm{p}_{1}\right) x=y \text { if and only if } p(x, x)=p(x, y)=p(y, y), \\
& \left(\mathrm{p}_{2}\right) p(x, x) \leq p(x, y) \\
& \left(\mathrm{p}_{3}\right) p(x, y)=p(y, x) \\
& \left(\mathrm{p}_{4}\right) p(x, y) \leq p(x, z)+p(z, y)-p(z, z) .
\end{aligned}
$$

A partial metric space is a pair $(X, p)$ such that $X$ is a nonempty set and $p$ is a partial metric on $X$. It is clear that if $p(x, y)=0$, then from $\left(\mathrm{p}_{1}\right)$ and $\left(\mathrm{p}_{2}\right) x=y$. But if $x=y$, $p(x, y)$ may not be 0 . A basic example of a partial metric space is the pair $\left(\mathbb{R}^{+}, p\right)$, where $p(x, y)=\max \{x, y\}$ for all $x, y \in \mathbb{R}^{+}$.

Lemma 3 (see [17]). Let $(X, d)$ and $(X, p)$ be a metric space and partial metric space, respectively. Then

(i) the function $\rho: X \times X \rightarrow \mathbb{R}^{+}$defined by $\rho(x, y)=$ $d(x, y)+p(x, y)$ is a partial metric;

(ii) let $\rho: X \times X \rightarrow \mathbb{R}^{+}$be defined by $\rho(x, y)=d(x, y)+$ $\max \{\omega(x), \omega(y)\}$; then $\rho$ is a partial metric on $X$, where $\omega: X \rightarrow \mathbb{R}^{+}$is an arbitrary function;

(iii) Let $\rho: \mathbb{R} \times \mathbb{R} \rightarrow \mathbb{R}$ be defined by $\rho(x, y)=\max \left\{2^{x}\right.$, $\left.2^{y}\right\}$; then $\rho$ is a partial metric on $\mathbb{R}$;

(iv) Let $\rho: X \times X \rightarrow \mathbb{R}^{+}$be defined by $\rho(x, y)=d(x, y)+a$; then $\rho$ is a partial metric on $X$, where $a \geq 0$.

Other examples of the partial metric spaces which are interesting from a computational point of view may be found in $[7,11,12,18]$.

Each partial metric $p$ on $X$ generates a $T_{0}$ topology $\tau_{p}$ on $X$ which has as a base the family of open $p$-balls $\left\{B_{p}(x, \varepsilon)\right.$ : $x \in X, \varepsilon>0\}$, where $B_{p}(x, \varepsilon)=\{y \in X: p(x, y)<p(x, x)+$ $\varepsilon\}$ for all $x \in X$ and $\varepsilon>0$.

Let $(X, p)$ be a partial metric.

A sequence $\left\{x_{n}\right\}$ in a partial metric space $(X, p)$ converges to a point $x \in X$ if and only if $p(x, x)=\lim _{n \rightarrow \infty} p\left(x, x_{n}\right)$.

A sequence $\left\{x_{n}\right\}$ in a partial metric space $(X, p)$ is called a Cauchy sequence if there exists (and is finite) $\lim _{n, m \rightarrow \infty} p\left(x_{n}, x_{m}\right)$.

A partial metric space $(X, p)$ is said to be complete if every Cauchy sequence $\left\{x_{n}\right\}$ in $X$ converges, with respect to $\tau_{p}$, to a point $x \in X$ such that $p(x, x)=\lim _{n, m \rightarrow \infty} p\left(x_{n}, x_{m}\right)$.

Suppose that $\left\{x_{n}\right\}$ is a sequence in partial metric space $(X, p)$; then we define $L\left(x_{n}\right)=\left\{x \mid x_{n} \rightarrow x\right\}$.

The following example shows that every convergent sequence $\left\{x_{n}\right\}$ in a partial metric space $(X, p)$ may not be a Cauchy sequence. In particular, it shows that the limit is not unique.
Example 4 (see [17]). Let $X=[0, \infty)$ and $p(x, y)=$ $\max \{x, y\}$. Let

$$
x_{n}= \begin{cases}0, & n=2 k \\ 1, & n=2 k+1 .\end{cases}
$$

Then clearly it is a convergent sequence, and for every $x \geq 1$ we have $\lim _{n \rightarrow \infty} p\left(x_{n}, x\right)=p(x, x)$, hence $L\left(x_{n}\right)=[1, \infty)$. But $\lim _{n, m \rightarrow \infty} p\left(x_{n}, x_{m}\right)$ does not exist; that is, it is not a Cauchy sequence.

Definition 5 (see [2]). A metric-like on a nonempty set $X$ is a mapping $\sigma: X \times X \rightarrow \mathbb{R}^{+}$such that for all $x, y, z \in X$,

$$
\begin{aligned}
& (\sigma 1) \sigma(x, y)=0 \Rightarrow x=y, \\
& (\sigma 2) \sigma(x, y)=\sigma(y, x), \\
& (\sigma 3) \sigma(x, y) \leq \sigma(x, z)+\sigma(z, y) .
\end{aligned}
$$

The pair $(X, \sigma)$ is called a metric-like space. Then a metric-like on $X$ satisfies all of the conditions of a metric except that $\sigma(x, x)$ may be positive for $x \in X$. Each metriclike $\sigma$ on $X$ generates a topology $\tau_{\sigma}$ on $X$ whose base is the family of open $\sigma$-ball, $\left\{B_{\sigma}(x, \varepsilon): x \in X, \varepsilon>0\right\}$, where $B_{\sigma}(x, \varepsilon)=\{y \in X:|\sigma(x, y)-\sigma(x, x)|<\varepsilon\}$ for all $x \in X$ and $\varepsilon>0$.

A sequence $\left\{x_{n}\right\}$ in a metric-like space $(X, \sigma)$ converges to a point $x \in X$ if and only if $\lim _{n \rightarrow \infty} \sigma\left(x, x_{n}\right)=\sigma(x, x)$.

A sequence $\left\{x_{n}\right\}$ in a metric-like space $(X, \sigma)$ is called a $\sigma$-Cauchy sequence if there exists (and is finite) $\lim _{n, m \rightarrow \infty} \sigma\left(x_{n}, x_{m}\right)$.

A metric-like space $(X, \sigma)$ is said to be complete if every $\sigma$-Cauchy sequence $\left\{x_{n}\right\}$ in $X$ converges, with respect to $\tau_{\sigma}$, to a point $x \in X$ such that

$$
\lim _{n \rightarrow \infty} \sigma\left(x_{n}, x\right)=\sigma(x, x)=\lim _{n, m \rightarrow \infty} \sigma\left(x_{n}, x_{m}\right) .
$$

Every partial metric space is a metric-like space. Below we give some examples of a metric-like space.

Example 6. Let $X=[0,1]$; then mapping $\sigma_{1}: X \times X \rightarrow \mathbb{R}^{+}$ defined by $\sigma_{1}(x, y)=x+y-x y$ is a metric-like on $X$.

Example 7. Let $X=\mathbb{R}$; then mappings $\sigma_{i}: X \times X \rightarrow \mathbb{R}^{+}(i \in$ $\{2,3,4\})$ defined by

$$
\begin{gathered}
\sigma_{2}(x, y)=|x|+|y|+a, \\
\sigma_{3}(x, y)=|x-b|+|y-b|, \\
\sigma_{4}(x, y)=x^{2}+y^{2}
\end{gathered}
$$

are metric-like space on $X$, where $a \geq 0$ and $b \in \mathbb{R}$.

\section{Main Results}

We start our work by proving the following crucial lemma.

Lemma 8. Let $(X, \sigma)$ be a metric-like space, and suppose that $\left\{x_{n}\right\}$ is $\sigma$-convergent to $x$. Then for every $y \in X$, one has

$$
\sigma(x, y)-\sigma(x, x) \leq \liminf _{n \rightarrow \infty} \sigma\left(x_{n}, y\right)
$$




$$
\begin{aligned}
& \leq \limsup _{n \rightarrow \infty} \sigma\left(x_{n}, y\right) \\
& \leq \sigma(x, y)+\sigma(x, x) .
\end{aligned}
$$

In particular, if $\sigma(x, x)=0$, then one has $\lim _{n \rightarrow \infty} \sigma\left(x_{n}, y\right)=$ $\sigma(x, y)$.

Proof. Using the triangle inequality in a metric-like space, it is easy to see that

$$
\begin{aligned}
& \sigma\left(x_{n}, y\right) \leq \sigma\left(x_{n}, x\right)+\sigma(x, y), \\
& \sigma(x, y) \leq \sigma\left(x, x_{n}\right)+\sigma\left(x_{n}, y\right) .
\end{aligned}
$$

Taking the upper limit as $n \rightarrow \infty$ in the first inequality and the lower limit as $n \rightarrow \infty$ in the second inequality, we obtain the desired result.

Theorem 9. Let $(X, \sigma)$ be a complete metric-like space. Let $T$ : $X \rightarrow X$ be a self-map, and let $\theta=:[0,1) \rightarrow(1 / 2,1]$ be defined by

$$
\theta(r)= \begin{cases}1, & 0 \leq r \leq \frac{\sqrt{5}-1}{2} \\ \frac{1-r}{r^{2}}, & \frac{\sqrt{5}-1}{2} \leq r \leq \frac{1}{\sqrt{2}} \\ \frac{1}{1+r}, & \frac{1}{\sqrt{2}} \leq r<1 .\end{cases}
$$

If there exists $r \in[0,1)$ such that for each $x, y \in X$

$$
\theta(r) \sigma(x, T x) \leq \sigma(x, y) \Longrightarrow \sigma(T x, T y) \leq r \sigma(x, y)
$$

Then $T$ has a unique fixed point $z \in X$, and for each $x \in X$, the sequence $\left\{T^{n} x\right\}$ converges to $z$.

Proof. Putting $y=T x$ in (10), hence from

$$
\theta(r) \sigma(x, T x) \leq \sigma(x, T x),
$$

it follows

$$
\sigma\left(T x, T^{2} x\right) \leq r \sigma(x, T x)
$$

for every $x \in X$. Let $x_{0} \in X$ be arbitrary and form the sequence $\left\{x_{n}\right\}$ by $x_{1}=T x_{0}$ and $x_{n+1}=T x_{n}$ for $n \in \mathbb{N} \cup\{0\}$. By (12), we have

$$
\begin{aligned}
\sigma\left(x_{n}, x_{n+1}\right) & =\sigma\left(T x_{n-1}, T^{2} x_{n-1}\right) \\
& \leq r \sigma\left(x_{n-1}, T x_{n-1}\right) \\
& =r \sigma\left(x_{n-1}, x_{n}\right) \\
& \vdots \\
& \leq r^{n} \sigma\left(x_{0}, x_{1}\right) .
\end{aligned}
$$

Also, by the condition $\sigma 3$ of the definition of metric-like space, for all $m \geq n$, we have

$$
\begin{aligned}
\sigma\left(x_{n}, x_{m}\right) \leq & \sigma\left(x_{n}, x_{n+1}\right) \\
& +\sigma\left(x_{n+1}, x_{n+2}\right) \\
& +\cdots+\sigma\left(x_{m-1}, x_{m}\right) \\
\leq & r^{n} \sigma\left(x_{0}, x_{1}\right)+r^{n+1} \sigma\left(x_{0}, x_{1}\right) \\
& +\cdots+r^{m-1} \sigma\left(x_{0}, x_{1}\right) \\
= & \frac{r^{n}-r^{m}}{1-r} \sigma\left(x_{0}, x_{1}\right) \\
< & \frac{r^{n}}{1-r} \sigma\left(x_{0}, x_{1}\right) \longrightarrow 0 \text { as } n \longrightarrow \infty .
\end{aligned}
$$

Hence, $\left\{x_{n}\right\}$ is a $\sigma$-Cauchy sequence.

Since $X$ is $\sigma$-complete, there exists $z \in X$ such that

$$
\lim _{n \rightarrow \infty} \sigma\left(x_{n}, z\right)=\sigma(z, z)=\lim _{m, n \rightarrow \infty} \sigma\left(x_{m}, x_{n}\right)=0 .
$$

That is, $\lim _{n \rightarrow \infty} x_{n+1}=\lim _{n \rightarrow \infty} T x_{n}=z$. We prove that $T z=$ $z$. Putting $x=T^{n-1} z$ in (12), we get that

$$
\sigma\left(T^{n} z, T^{n+1} z\right) \leq r \sigma\left(T^{n-1} z, T^{n} z\right)
$$

holds for each $n \in \mathbb{N}$ (where $T^{0} z=z$ ). It follows by induction that

$$
\sigma\left(T^{n} z, T^{n+1} z\right) \leq r^{n} \sigma(z, T z)
$$

Let us prove now that

$$
\sigma(z, T x) \leq r \sigma(z, x)
$$

holds for each $x \neq z$. Since $\sigma\left(x_{n}, T x_{n}\right) \rightarrow 0$ and by Lemma $8 \sigma\left(x_{n}, x\right) \rightarrow \sigma(z, x) \neq 0$, it follows that there exists $n_{0} \in \mathbb{N}$ such that

$$
\theta(r) \sigma\left(x_{n}, T x_{n}\right) \leq \sigma\left(x_{n}, x\right)
$$

holds for every $n \geq n_{0}$. Assumption (10) implies that for such $n \sigma\left(T x_{n}, T x\right) \leq r \sigma\left(x_{n}, x\right)$, thus as $n \rightarrow \infty$, we get that

$$
\sigma(z, T x) \leq r \sigma(z, x) .
$$

We will prove that

$$
\sigma\left(T^{n} z, z\right) \leq \sigma(T z, z),
$$

for each $n \in \mathbb{N}$. For $n=1$, this relation is obvious. Suppose that it holds for some $m \in \mathbb{N}$. If $T^{m} z=z$, then $T^{m+1} z=$ $T z$ and $\sigma\left(T^{m+1} z, z\right)=\sigma(T z, z) \leq \sigma(T z, z)$. If $T^{m} z \neq z$, then applying (18) and the induction hypothesis; we get that

$$
\begin{aligned}
\sigma\left(T^{m+1} z, z\right) & \leq r \sigma\left(T^{m} z, z\right) \\
& \leq r \sigma(T z, z) \leq \sigma(T z, z),
\end{aligned}
$$

and (21) is proved by induction. 
In order to prove that $T z=z$, we consider two possible cases.

Case I. $0 \leq r<1 / \sqrt{2}$ (and hence $\theta(r) \leq(1-r) / r^{2}$ ). We will prove first that

$$
\sigma\left(T^{n} z, T z\right) \leq r \sigma(T z, z)
$$

for $n \geq 2$. For $n=2$, it follows from (16). Suppose that (23) holds for some $n>2$. Then

$$
\begin{aligned}
\sigma(T z, z) & \leq \sigma\left(z, T^{n} z\right)+\sigma\left(T^{n} z, T z\right) \\
& \leq \sigma\left(z, T^{n} z\right)+r \sigma(z, T z)
\end{aligned}
$$

which implies $(1-r) \sigma(z, T z) \leq \sigma\left(z, T^{n} z\right)$. Using (17) we obtain

$$
\begin{aligned}
\theta(r) \sigma\left(T^{n} z, T^{n+1} z\right) & \leq \frac{1-r}{r^{n}} \sigma\left(T^{n} z, T^{n+1} z\right) \\
& \leq \frac{1-r}{r^{n}} \cdot r^{n} \sigma(z, T z) \\
& =(1-r) \sigma(z, T z) \leq \sigma\left(z, T^{n} z\right)
\end{aligned}
$$

Assumption (10) and relation (21) imply that

$$
\begin{aligned}
\sigma\left(T z, T^{n+1} z\right) & \leq r \sigma\left(z, T^{n} z\right) \\
& \leq r \sigma(z, T z)
\end{aligned}
$$

So relation (23) is proved by induction.

Now $T z \neq z$ and (23) implies that $T^{n} z \neq z$ for each $n \in \mathbb{N}$. Hence, (18) imply that

$$
\begin{aligned}
\sigma\left(z, T^{n+1} z\right) & \leq r \sigma\left(z, T^{n} z\right) \\
& \leq r^{2} \sigma\left(z, T^{n-1} z\right) \\
& \leq r^{n} \sigma(z, T z)
\end{aligned}
$$

Hence $\lim _{n \rightarrow \infty} \sigma\left(z, T^{n+1} z\right)=0=\sigma(z, z)$, thus $T^{n} z \rightarrow z$ and; using Lemma 8 in (23), we have $\sigma(z, T z) \leq r \sigma(T z, z)$ as $n \rightarrow \infty$ which implies that $\sigma(z, T z)=0$, a contradiction.

Case II. $1 / \sqrt{2} \leq r<1$ (and so $\theta(r)=1 /(1+r)$ ). We will prove that there exists a subsequence $\left\{x_{n_{k}}\right\}$ of $\left\{x_{n}\right\}$ such that

$$
\theta(r) \sigma\left(x_{n_{k}}, T x_{n_{k}}\right)=\theta(r) \sigma\left(x_{n_{k}}, x_{n_{k}+1}\right) \leq \sigma\left(x_{n_{k}}, z\right)
$$

holds for each $k \in \mathbb{N}$. From (12) we know that $\sigma\left(x_{n}, x_{n+1}\right) \leq$ $r \sigma\left(x_{n-1}, x_{n}\right)$ holds for each $n \in \mathbb{N}$. Suppose that

$$
\begin{gathered}
\frac{1}{1+r} \sigma\left(x_{n-1}, x_{n}\right)>\sigma\left(x_{n-1}, z\right), \\
\frac{1}{1+r} \sigma\left(x_{n}, x_{n+1}\right)>\sigma\left(x_{n}, z\right)
\end{gathered}
$$

hold for some $n \in \mathbb{N}$. Then

$$
\begin{aligned}
\sigma\left(x_{n-1}, x_{n}\right) & \leq \sigma\left(x_{n-1}, z\right)+\sigma\left(z, x_{n}\right) \\
& <\frac{1}{1+r} \sigma\left(x_{n-1}, x_{n}\right)+\sigma\left(x_{n}, z\right) \\
& <\frac{1}{1+r} \sigma\left(x_{n-1}, x_{n}\right)+\frac{1}{1+r} \sigma\left(x_{n}, x_{n+1}\right) \\
& \leq \frac{1}{1+r} \sigma\left(x_{n-1}, x_{n}\right)+\frac{r}{1+r} \sigma\left(x_{n-1}, x_{n}\right) \\
& =\sigma\left(x_{n-1}, x_{n}\right),
\end{aligned}
$$

which is impossible. Hence one of the following holds for each $n$ :

$$
\theta(r) \sigma\left(x_{n-1}, x_{n}\right) \leq \sigma\left(x_{n-1}, z\right)
$$

or

$$
\theta(r) \sigma\left(x_{n}, x_{n+1}\right) \leq \sigma\left(x_{n}, z\right)
$$

In particular,

$$
\theta(r) \sigma\left(x_{2 n-1}, x_{2 n}\right) \leq \sigma\left(x_{2 n-1}, z\right),
$$

or

$$
\theta(r) \sigma\left(x_{2 n}, x_{2 n+1}\right) \leq \sigma\left(x_{2 n}, z\right) .
$$

In other words, there is a subsequence $\left\{x_{n_{k}}\right\}$ of $\left\{x_{n}\right\}$ such that (28) holds for each $k \in \mathbb{N}$. But then assumption (10) implies that

$$
\sigma\left(T x_{n_{k}}, T z\right) \leq r \sigma\left(x_{n_{k}}, z\right)
$$

or

$$
\sigma\left(T x_{n_{k-1}}, T z\right) \leq r \sigma\left(x_{n_{k-1}}, z\right) .
$$

Passing to the limit when $k \rightarrow \infty$, we get that $\sigma(z, T z) \leq 0$, which is possible only if $T z=z$.

Thus, we have proved that $z$ is a fixed point of $T$. The uniqueness of the fixed point follows easily from (10). Indeed, if $y$ and $z$ are two fixed points of $T$ such that $y \neq z$, then from (18) we have

$$
\begin{aligned}
\sigma(y, z) & =\sigma(y, T z) \\
& \leq r \sigma(y, z),
\end{aligned}
$$

which is a contradiction.

According to Theorem 9, we get the following result.

Corollary 10 (see [19]). Let $(X, d)$ be a complete metric space, and let $T$ be a mapping on $X$. Define a nonincreasing function $\theta$ from $[0,1]$ into $[1 / 2,1]$ by

$$
\theta(r)= \begin{cases}1, & 0 \leq r \leq \frac{\sqrt{5}-1}{2}, \\ \frac{1-r}{r^{2}}, & \frac{\sqrt{5}-1}{2} \leq r \leq \frac{1}{\sqrt{2}} \\ \frac{1}{1+r}, & \frac{1}{\sqrt{2}} \leq r<1 .\end{cases}
$$


Assume that there exists $r \in[0,1]$, such that

$$
\theta(r) d(x, T x) \leq d(x, y) \Longrightarrow d(T x, T y) \leq r d(x, y),
$$

for all $x, y \in X$; then there exists a unique fixed-point $z$ of $T$. Moreover, $\lim _{n \rightarrow \infty} T^{n} x=z$ for all $x \in X$.

Proof. Using a similar argument given in Theorem 9 for $\sigma(x, y)=d(x, y)$, the desired result is obtained.

Now, in order to support the useability of our results, let us introduce the following example.

Example 11. Let $X=[0, \infty)$. Define $\sigma: X \times X \rightarrow \mathbb{R}^{+}$by

$$
\sigma(x, y)=x+y
$$

for all $x, y \in X$. Then $(X, \sigma)$ is a complete metric-like space. Define a map $T: X \rightarrow X$ by

$$
T(x)=\ln \left(1+\frac{1}{\sqrt{2}} x\right)
$$

for $x \in X$. Then for each $x, y \in X$, we have

$$
\begin{aligned}
\frac{1}{1+1 / \sqrt{2}} \sigma(x, T x) & =\frac{\sqrt{2}}{\sqrt{2}+1}\left(x+\ln \left(1+\frac{1}{\sqrt{2}} x\right)\right) \\
& \leq \frac{\sqrt{2}}{\sqrt{2}+1}\left(x+\frac{1}{\sqrt{2}} x\right)=x \\
& \leq x+y=\sigma(x, y) .
\end{aligned}
$$

On the other hand, we have

$$
\begin{aligned}
\sigma(T x, T y) & =\ln \left(1+\frac{1}{\sqrt{2}} x\right)+\ln \left(1+\frac{1}{\sqrt{2}} y\right) \\
& \leq \frac{1}{\sqrt{2}} x+\frac{1}{\sqrt{2}} y \\
& =\frac{1}{\sqrt{2}} \sigma(x, y) .
\end{aligned}
$$

Thus $T$ satisfies all the hypotheses of Theorem 9 , and hence $T$ has a unique fixed point. Indeed, $r=1 / \sqrt{2}, \theta(r)=1 /(1+r)$, and 0 is the unique fixed point of $T$.

Theorem 12. Let $(X, \sigma)$ be a complete metric-like space. Let $S$, $T: X \rightarrow X$ be two self-mappings. Suppose that there exists $r \in[0,1)$ such that

$$
\begin{array}{r}
\max \{\sigma(S(x), T S(x)), \sigma(T(x), S T(x))\} \\
\leq r \min \{\sigma(x, S(x)), \sigma(x, T(x))\}
\end{array}
$$

for every $x \in X$ and that

$$
\begin{gathered}
\alpha(y)=\inf \{\sigma(x, y)+\min \{\sigma(x, S(x)), \sigma(x, T(x))\}: \\
x \in X\}>0
\end{gathered}
$$

for every $y \in X$ with $y$ that is not a common fixed point of $S$ and $T$. Then there exists $z \in X$ such that $z=S(z)=T(z)$. Moreover, if $v=S(v)=T(v)$, then $\sigma(v, v)=0$.
Proof. Let $x_{0} \in X$ be arbitrary, and define a sequence $\left\{x_{n}\right\}$ by

$$
\begin{aligned}
x_{n} & =S\left(x_{n-1}\right), \quad \text { if } n \text { is odd } \\
& =T\left(x_{n-1}\right), \quad \text { if } n \text { is even. }
\end{aligned}
$$

Then if $n \in \mathbb{N}$ is odd, we have

$$
\begin{aligned}
& \sigma\left(x_{n}, x_{n+1}\right) \\
& =\sigma\left(S\left(x_{n-1}\right), T\left(x_{n}\right)\right) \\
& =\sigma\left(S\left(x_{n-1}\right), T S\left(x_{n-1}\right)\right) \\
& \leq \max \left\{\sigma\left(S\left(x_{n-1}\right), T S\left(x_{n-1}\right)\right),\right. \\
& \left.\quad \sigma\left(T\left(x_{n-1}\right), S T\left(x_{n-1}\right)\right)\right\} \\
& \leq r \min \left\{\sigma\left(x_{n-1}, S\left(x_{n-1}\right)\right), \sigma\left(x_{n-1}, T\left(x_{n-1}\right)\right)\right\} \\
& \leq \operatorname{r\sigma }\left(x_{n-1}, S\left(x_{n-1}\right)\right) \\
& =\operatorname{r\sigma }\left(x_{n-1}, x_{n}\right) .
\end{aligned}
$$

If $n$ is even, then by (44), we have

$$
\begin{aligned}
& \sigma\left(x_{n}, x_{n+1}\right) \\
&= \sigma\left(T\left(x_{n-1}\right), S\left(x_{n}\right)\right) \\
&=\sigma\left(T\left(x_{n-1}\right), S T\left(x_{n-1}\right)\right) \\
& \leq \max \left\{\sigma\left(T\left(x_{n-1}\right), S T\left(x_{n-1}\right)\right),\right. \\
&\left.\sigma\left(S\left(x_{n-1}\right), T S\left(x_{n-1}\right)\right)\right\} \\
& \leq r \min \left\{\sigma\left(x_{n-1}, T\left(x_{n-1}\right)\right), \sigma\left(x_{n-1}, S\left(x_{n-1}\right)\right)\right\} \\
& \leq r \sigma\left(x_{n-1}, T\left(x_{n-1}\right)\right) \\
&= \operatorname{r\sigma } \sigma\left(x_{n-1}, x_{n}\right) .
\end{aligned}
$$

Thus for any positive integer $n$, it must be the case that

$$
\sigma\left(x_{n}, x_{n+1}\right) \leq r \sigma\left(x_{n-1}, x_{n}\right) .
$$

Repeating (49), we obtain

$$
\sigma\left(x_{n}, x_{n+1}\right) \leq r^{n} \sigma\left(x_{0}, x_{1}\right) .
$$

So, if $m>n$, then

$$
\begin{aligned}
\sigma\left(x_{n}, x_{m}\right) \leq & \sigma\left(x_{n}, x_{n+1}\right) \\
& +\sigma\left(x_{n+1}, x_{n+2}\right)+\cdots+\sigma\left(x_{m-1}, x_{m}\right) \\
\leq & {\left[r^{n}+r^{n+1}+\cdots+r^{m-1}\right] \sigma\left(x_{0}, x_{1}\right) } \\
\leq & \frac{r^{n}}{1-r} \sigma\left(x_{0}, x_{1}\right) .
\end{aligned}
$$

Thus $\lim _{n, m \rightarrow \infty} \sigma\left(x_{n}, x_{m}\right)=0$.

That is, $\left\{x_{n}\right\}$ is a $\sigma$-Cauchy sequence in the metric-like space $(X, \sigma)$. Since $(X, \sigma)$ is $\sigma$-complete, there exist $z \in X$ such that

$$
\sigma(z, z)=\lim _{n \rightarrow \infty} \sigma\left(x_{n}, z\right)=\lim _{n, m \rightarrow \infty} \sigma\left(x_{n}, x_{m}\right)=0 .
$$


Assume that $z$ is not a common fixed point of $S$ and $T$. Then by hypothesis

$$
\begin{aligned}
0 & <\inf \{\sigma(x, z)+\min \{\sigma(x, S(x)), \sigma(x, T(x))\}: x \in X\} \\
\leq & \inf \left\{\sigma\left(x_{n}, z\right)+\min \left\{\sigma\left(x_{n}, S\left(x_{n}\right)\right), \sigma\left(x_{n}, T\left(x_{n}\right)\right)\right\}:\right. \\
& n \in \mathbb{N}\} \\
& \leq \inf \left\{\frac{r^{n}}{1-r} \sigma\left(x_{0}, x_{1}\right)+\sigma\left(x_{n}, x_{n+1}\right): n \in \mathbb{N}\right\} \\
& \leq \inf \left\{\frac{r^{n}}{1-r} \sigma\left(x_{0}, x_{1}\right)+r^{n} \sigma\left(x_{0}, x_{1}\right): n \in \mathbb{N}\right\}=0
\end{aligned}
$$

which is a contradiction. Therefore, $z=S(z)=T(z)$.

If $v=S(v)=T(v)$ for some $v \in X$, then

$$
\begin{aligned}
\sigma(v, v) & =\max \{\sigma(S(v), T S(v)), \sigma(T(v), S T(v))\} \\
& \leq r \min \{\sigma(v, S(v)), \sigma(v, T(v))\} \\
& =r \min \{\sigma(v, v), \sigma(v, v)\} \\
& =r \sigma(v, v)
\end{aligned}
$$

which gives that $\sigma(v, v)=0$.

Example 13. Let $(X, \sigma)$ be a metric-like space where $X=$ $\{1 / n\}_{n=1}^{\infty} \cup\{0\}$ and $\sigma(x, y)=x+y$. Define $S: X \rightarrow X$ by $S(0)=0, S(1 / 2 n)=1 /(4 n+3)$, and $S(1 /(2 n-1))=0$ and $T(0)=0, T(1 /(2 n-1))=1 /(4 n+4)$, and $T(1 / 2 n)=0$. Then for $x=1 / 2 n$, we have

$$
\begin{aligned}
\max \{\sigma(S(x), T S(x)), \sigma(T(x), S T(x))\} \\
=\max \left\{\sigma\left(S\left(\frac{1}{2 n}\right), T\left(S\left(\frac{1}{2 n}\right)\right)\right),\right. \\
=\max \left\{\frac{1}{4 n+3}+\frac{1}{8 n+12}, 0\right\}=\frac{1}{4 n+3}+\frac{1}{8 n+12} \\
\leq r \min \{\sigma(x, S(x)), \sigma(x, T(x))\} \\
=r \min \left\{\frac{1}{2 n}+\frac{1}{4 n+3}, \frac{1}{2 n}+0\right\}=r \frac{1}{2 n} .
\end{aligned}
$$

It is easy to see that the above inequality is true for $x=1 /(2 n-$ 1 ) and for $3 / 4 \leq r<1$. Also,

$$
\begin{gathered}
\alpha(y)=\inf \{\sigma(x, y)+\min \{\sigma(x, S(x)), \sigma(x, T(x))\}: \\
x \in X\}>0
\end{gathered}
$$

for every $y \in X$ with y is not a common fixed point of $S$ and $T$. This shows that all conditions of Theorem 12 are satisfied and 0 is a common fixed point for $S$ and $T$.
Corollary 14. Let $(X, \sigma)$ be a complete metric-like space, and let $T: X \rightarrow X$ be a mapping. Suppose that there exists $r \in$ $[0,1)$ such that

$$
\sigma\left(T(x), T^{2}(x)\right) \leq r \sigma(x, T(x))
$$

for every $x \in X$ and that

$$
\alpha(y)=\inf \{\sigma(x, y)+\sigma(x, T(x)): x \in X\}>0
$$

for every $y \in X$ with $y \neq T(y)$. Then there exists $z \in X$ such that $z=T(z)$. Moreover, if $v=T(v)$, then $\sigma(v, v)=0$.

Proof. Taking $S=T$ in Theorem 12, the conclusion of the corollary follows.

Theorem 15. Let $(X, \sigma)$ be a complete metric-like space. Let $S, T$ be mappings from $X$ onto itself. Suppose that there exists $r>1$ such that

$$
\begin{array}{r}
\min \{\sigma(T S(x), S(x)), \sigma(S T(x), T(x))\} \\
\geq r \max \{\sigma(S x, x), \sigma(T x, x)\}
\end{array}
$$

for every $x \in X$ and that

$$
\begin{gathered}
\alpha(y)=\inf \{\sigma(x, y)+\min \{\sigma(x, S(x)), \sigma(x, T(x))\}: \\
x \in X\}>0
\end{gathered}
$$

for every $y \in X$ with $y$ that is not a common fixed point of $S$ and $T$. Then there exists $z \in X$ such that $z=S(z)=T(z)$. Moreover, if $v=S(v)=T(v)$, then $\sigma(v, v)=0$.

Proof. Let $x_{0} \in X$ be arbitrary. Since $S$ is onto, there is an element $x_{1}$ satisfying $x_{1} \in S^{-1}\left(x_{0}\right)$. Since $T$ is also onto, there is an element $x_{2}$ satisfying $x_{2} \in T^{-1}\left(x_{1}\right)$. Proceeding in the same way, we can find that $x_{2 n+1} \in S^{-1}\left(x_{2 n}\right)$ and $x_{2 n+2} \in$ $T^{-1}\left(x_{2 n+1}\right)$ for $n=1,2,3, \ldots$. Therefore, $x_{2 n}=S x_{2 n+1}$ and $x_{2 n+1}=T x_{2 n+2}$ for $n=0,1,2, \ldots$ If $n=2 m$, then using (59)

$$
\begin{aligned}
& \sigma\left(x_{n-1}, x_{n}\right) \\
&= \sigma\left(x_{2 m-1}, x_{2 m}\right) \\
&=\sigma\left(T x_{2 m}, S x_{2 m+1}\right) \\
&=\sigma\left(T S x_{2 m+1}, S x_{2 m+1}\right) \\
& \geq \min \left\{\sigma\left(T S\left(x_{2 m+1}\right), S\left(x_{2 m+1}\right)\right),\right. \\
& \geq \operatorname{rmax}\left\{\sigma\left(S T\left(x_{2 m+1}\right), T\left(x_{2 m+1}\right)\right)\right\} \\
& \geq\left.\operatorname{r\sigma }\left(S x_{2 m+1}, x_{2 m+1}\right), \sigma\left(T x_{2 m+1}, x_{2 m+1}\right)\right\} \\
&= \operatorname{r\sigma }\left(x_{2 m}, x_{2 m+1}\right) \\
&= \operatorname{r\sigma }\left(x_{n}, x_{n+1}\right) .
\end{aligned}
$$




$$
\begin{aligned}
& \text { If } n=2 m+1 \text {, then using (59) } \\
& \begin{aligned}
\sigma\left(x_{n-1}, x_{n}\right) \\
=\sigma\left(x_{2 m}, x_{2 m+1}\right) \\
=\sigma\left(S x_{2 m+1}, T x_{2 m+2}\right) \\
=\sigma\left(S T x_{2 m+2}, T x_{2 m+2}\right) \\
\geq \min \left\{\sigma\left(T S\left(x_{2 m+2}\right), S\left(x_{2 m+2}\right)\right),\right. \\
\left.\quad \sigma\left(S T\left(x_{2 m+2}\right), T\left(x_{2 m+2}\right)\right)\right\} \\
\geq r \max \left\{\sigma\left(S x_{2 m+2}, x_{2 m+2}\right), \sigma\left(T x_{2 m+2}, x_{2 m+2}\right)\right\} \\
\geq r \sigma\left(T x_{2 m+2}, x_{2 m+2}\right) \\
=r \sigma\left(x_{2 m+1}, x_{2 m+2}\right) \\
=r \sigma\left(x_{n}, x_{n+1}\right) .
\end{aligned}
\end{aligned}
$$

Thus for any positive integer $n$, it must be the case that

$$
\sigma\left(x_{n-1}, x_{n}\right) \geq \operatorname{ro}\left(x_{n}, x_{n+1}\right)
$$

which implies that

$$
\sigma\left(x_{n}, x_{n+1}\right) \leq \frac{1}{r} \sigma\left(x_{n-1}, x_{n}\right) \leq \cdots \leq\left(\frac{1}{r}\right)^{n} \sigma\left(x_{0}, x_{1}\right) .
$$

Let $\alpha=1 / r$; then $0<\alpha<1$ since $r>1$.

Now, (64) becomes

$$
\sigma\left(x_{n}, x_{n+1}\right) \leq \alpha^{n} \sigma\left(x_{0}, x_{1}\right) .
$$

So, if $m>n$, then

$$
\begin{aligned}
\sigma\left(x_{n}, x_{m}\right) \leq & \sigma\left(x_{n}, x_{n+1}\right) \\
& +\sigma\left(x_{n+1}, x_{n+2}\right)+\cdots+\sigma\left(x_{m-1}, x_{m}\right) \\
\leq & {\left[\alpha^{n}+\alpha^{n+1}+\cdots+\alpha^{m-1}\right] \sigma\left(x_{0}, x_{1}\right) } \\
\leq & \frac{\alpha^{n}}{1-\alpha} \sigma\left(x_{0}, x_{1}\right) .
\end{aligned}
$$

Thus $\lim _{n, m \rightarrow \infty} \sigma\left(x_{n}, x_{m}\right)=0$. That is, $\left\{x_{n}\right\}$ is a $\sigma$-Cauchy sequence in the metric-like space $(X, \sigma)$. Since $(X, \sigma)$ is $\sigma$ complete, there exists $z \in X$ such that

$$
\sigma(z, z)=\lim _{n \rightarrow \infty} \sigma\left(x_{n}, z\right)=\lim _{n, m \rightarrow \infty} \sigma\left(x_{n}, x_{m}\right)=0 .
$$

Assume that $z$ is not a common fixed point of $S$ and $T$. Then by hypothesis

$$
\begin{aligned}
0 & <\inf \{\sigma(x, z)+\min \{\sigma(x, S(x)), \sigma(x, T(x))\}: x \in X\} \\
\leq & \inf \left\{\sigma\left(x_{n}, z\right)+\min \left\{\sigma\left(x_{n}, S\left(x_{n}\right)\right), \sigma\left(x_{n}, T\left(x_{n}\right)\right)\right\}:\right. \\
& n \in \mathbb{N}\} \\
& \leq \inf \left\{\frac{\alpha^{n}}{1-\alpha} \sigma\left(x_{0}, x_{1}\right)+\sigma\left(x_{n-1}, x_{n}\right): n \in \mathbb{N}\right\} \\
& \leq \inf \left\{\frac{\alpha^{n}}{1-\alpha} \sigma\left(x_{0}, x_{1}\right)+\alpha^{n-1} \sigma\left(x_{0}, x_{1}\right): n \in \mathbb{N}\right\}=0
\end{aligned}
$$

which is a contradiction. Therefore, $z=S(z)=T(z)$.

$$
\begin{aligned}
& \text { If } v=S(v)=T(v) \text { for some } v \in X \text {, then } \\
& \begin{aligned}
\sigma(v, v) & =\min \{\sigma(T S(v), S(v)), \sigma(S T(v), T(v))\} \\
& \geq r \max \{\sigma(S(v), v), \sigma(T(v), v)\} \\
& =r \max \{\sigma(v, v), \sigma(v, v)\} \\
& =r \sigma(v, v)
\end{aligned}
\end{aligned}
$$

which gives that $\sigma(v, v)=0$.

Corollary 16. Let $(X, \sigma)$ be a complete metric-like space, and let $T: X \rightarrow X$ be an onto mapping. Suppose that there exists $r \in[0,1)$ such that

$$
\sigma\left(T^{2}(x), T(x)\right) \geq \operatorname{ro}(T(x), x)
$$

for every $x \in X$ and that

$$
\alpha(y)=\inf \{\sigma(x, y)+\sigma(T(x), x): x \in X\}>0
$$

for every $y \in X$ with $y \neq T(y)$. Then there exists $z \in X$ such that $z=T(z)$. Moreover, if $v=T(v)$, then $\sigma(v, v)=0$.

Proof. Taking $S=T$ in Theorem 15, we have the desired result.

Definition 17. Let $(X, \sigma)$ and $(Y, \tau)$ be metric-like spaces. Then $f: X \rightarrow Y$ is said to be a continuous mapping, if $\lim _{n \rightarrow \infty} x_{n}=x$ implies that $\lim _{n \rightarrow \infty} f\left(x_{n}\right)=f(x)$.

Corollary 18. Let $(X, \sigma)$ be a complete metric-like space, and let $T$ be a mapping of $X$ into itself. If there is a real number $r$ with $r>1$ satisfying

$$
\sigma\left(T^{2}(x), T(x)\right) \geq \operatorname{ro}(T(x), x)
$$

for every $x \in X$ and $T$ is onto and continuous, then $T$ has $a$ fixed point.

Proof. Assume that there exists $y \in X$ with $y \neq T(y)$ and

$$
\inf \{\sigma(x, y)+\sigma(T(x), x): x \in X\}=0 \text {. }
$$

Then there exists a sequence $\left\{x_{n}\right\}$ such that

$$
\lim _{n \rightarrow \infty}\left\{\sigma\left(x_{n}, y\right)+\sigma\left(T\left(x_{n}\right), x_{n}\right)\right\}=0
$$

So, we have $\sigma\left(x_{n}, y\right) \rightarrow 0$ and $\sigma\left(T\left(x_{n}\right), x_{n}\right) \rightarrow 0$ as $n \rightarrow \infty$. Since, $\sigma(y, y) \leq \sigma\left(y, x_{n}\right)+\sigma\left(x_{n}, y\right)$, hence $\sigma(y, y) \rightarrow 0$ as $n \rightarrow \infty$. Now,

$$
\begin{array}{r}
\sigma\left(T\left(x_{n}\right), y\right) \leq \sigma\left(T\left(x_{n}\right), x_{n}\right)+\sigma\left(x_{n}, y\right) \longrightarrow 0 \\
\text { as } n \longrightarrow \infty .
\end{array}
$$

Since $T$ is continuous, we have

$$
T(y)=T\left(\lim _{n \rightarrow \infty} x_{n}\right)=\lim _{n \rightarrow \infty} T\left(x_{n}\right)=y .
$$

This is a contradiction. Hence if $y \neq T(y)$, then

$$
\inf \{\sigma(x, y)+\sigma(T(x), x): x \in X\}>0 \text {, }
$$

which is condition (71) of Corollary 16. By Corollary 16, there exists $z \in X$ such that $z=T(z)$. 
Now we give an example to support our result.

Example 19. Let $X=[0, \infty)$ and $\sigma(x, y)=x+y$. Define $T$ : $X \rightarrow X$ by $T(x)=2 x$.

Obviously $T$ is onto and continuous. Also for each $x, y \in$ $X$, we have

$$
\sigma\left(T^{2} x, T x\right)=4 x+2 x=6 x \geq r 3 x=r \sigma(T x, x),
$$

where $r=2$. Thus $T$ satisfies the conditions given in Corollary 18, and 0 is the fixed point of $T$.

Corollary 20. Let $(X, \sigma)$ be a complete metric-like space, and $T$ be a mapping of $X$ into itself. If there is a real number $r$ with $r>1$ satisfying

$\sigma(T(x), T(y)) \geq r \min \{\sigma(x, T(x)), \sigma(T(y), y), \sigma(x, y)\}$

for every $x, y \in X$ and $T$ is onto and continuous, then $T$ has a fixed point.

Proof. Replacing $y$ by $T(x)$ in (79), we obtain

$$
\begin{aligned}
& \sigma\left(T(x), T^{2}(x)\right) \\
& \quad \geq r \min \left\{\sigma(x, T(x)), \sigma\left(T^{2}(x), T(x)\right), \sigma(x, T(x))\right\}
\end{aligned}
$$

for all $x \in X$.

Without loss of generality, we may assume that $T(x) \neq$ $T^{2}(x)$. Otherwise $T$ has a fixed point. Since $r>1$, it follows from $(80)$ that

$$
\sigma\left(T^{2}(x), T(x)\right) \geq r \sigma(T(x), x)
$$

for every $x \in X$. By the argument similar to that used in Corollary 18 , we can prove that if $y \neq T(y)$, then

$$
\inf \{\sigma(x, y)+\sigma(T(x), x): x \in X\}>0,
$$

which is condition (71) of Corollary 16. So, Corollary 16 applies to obtain a fixed point of $T$.

According to Theorem 12, we get the following result.

Corollary 21 (see [17, Theorem 1]). Let $(X, p)$ be a complete partial metric space. Let $S, T: X \rightarrow X$ be two self-mappings. Suppose that there exists $r \in[0,1)$ such that

$$
\begin{aligned}
\max & \{p(S(x), T S(x)), p(T(x), S T(x))\} \\
& \leq r \min \{p(x, S(x)), p(x, T(x))\}
\end{aligned}
$$

for every $x \in X$ and that

$$
\begin{gathered}
\alpha(y)=\inf \{p(x, y)+\min \{p(x, S(x)), p(x, T(x))\}: \\
x \in X\}>0
\end{gathered}
$$

for every $y \in X$ with $y$ that is not a common fixed point of $S$ and $T$. Then there exists $z \in X$ such that $z=S(z)=T(z)$. Moreover, if $v=S(v)=T(v)$, then $p(v, v)=0$.
Proof. Using a similar argument given in the Theorem 12 for $\sigma(x, y)=p(x, y)$, the desired result is obtained, where $p$ is a partial metric on $X$.

Also, according to Theorem 15, we get Theorem 2 from [17].

\section{References}

[1] A. Aghajani, S. Radenović, and J. R. Roshan, "Common fixed point results for four mappings satisfying almost generalized $(S, T)$-contractive condition in partially ordered metric spaces," Applied Mathematics and Computation, vol. 218, no. 9, pp. 5665$5670,2012$.

[2] A. Amini-Harandi, "Metric-like spaces, partial metric spaces and fixed points," Fixed Point Theory and Applications, vol. 2012, article 204, 10 pages, 2012.

[3] D. Đorić, Z. Kadelburg, and S. Radenović, "Edelstein-Suzukitype fixed point results in metric and abstract metric spaces," Nonlinear Analysis: Theory, Methods \& Applications, vol. 75, no. 4, pp. 1927-1932, 2012.

[4] N. Hussain, D. Đorić, Z. Kadelburg, and S. Radenović, "Suzukitype fixed point results in metric type spaces," Fixed Point Theory and Applications, vol. 2012, article 126, 12 pages, 2012.

[5] N. Hussain and M. H. Shah, "KKM mappings in cone $b$-metric spaces," Computers \& Mathematics with Applications, vol. 62, no. 4, pp. 1677-1684, 2011.

[6] N. Hussain, M. H. Shah, A. Amini-Harandi, and Z. Akhtar, "Common fixed point theorems for generalized contractive mappings with applications," Fixed Point Theory and Applications, vol. 2013, article 169, 17 pages, 2013.

[7] N. Hussain, Z. Kadelburg, S. Radenović, and F. Al-Solamy, "Comparison functions and fixed point results in partial metric spaces," Abstract and Applied Analysis, vol. 2012, Article ID 605781, 15 pages, 2012.

[8] M. Kikkawa and T. Suzuki, "Some similarity between contractions and Kannan mappings," Fixed Point Theory and Applications, vol. 2008, Article ID 649749, 8 pages, 2008.

[9] M. Kikkawa and T. Suzuki, "Some notes on fixed point theorems with constants," Bulletin of the Kyushu Institute of Technology, no. 56, pp. 11-18, 2009.

[10] M. Kikkawa and T. Suzuki, "Three fixed point theorems for generalized contractions with constants in complete metric spaces," Nonlinear Analysis: Theory, Methods \& Applications, vol. 69, no. 9, pp. 2942-2949, 2008.

[11] M. A. Kutbi, J. Ahmad, N. Hussain, and M. Arshad, "Common fixed point results for mappings with rational expressions," Abstract and Applied Analysis, vol. 2013, Article ID 549518, 11 pages, 2013.

[12] S. G. Matthews, "Partial metric topology," in Papers on General Topology and Applications, vol. 728 of Annals of the New York Academy of Sciences, pp. 183-197, New York Academy of Sciences, New York, NY, USA, 1994.

[13] V. Parvaneh, J. R. Roshan, and S. Radenović, "Existence of tripled coincidence points in ordered b-metric spaces and an application to a system of integral equations," Fixed Point Theory and Applications, vol. 2013, article 130, 19 pages, 2013.

[14] O. Popescu, "Fixed point theorem in metric spaces," Bulletin of the Transilvania University of Braşov, vol. 150, pp. 479-482, 2008. 
[15] O. Popescu, "Two fixed point theorems for generalized contractions with constants in complete metric space," Central European Journal of Mathematics, vol. 7, no. 3, pp. 529-538, 2009.

[16] J. R. Roshan, V. Parvaneh, S. Sedghi, N. Shobkolaei, and W. Shatanawi, "Common fixed points of almost generalized $(\psi, \varphi)_{s}$-contractive mappings in ordered $b$-metric spaces," Fixed Point Theory and Applications, vol. 2013, article 159, 23 pages, 2013.

[17] S. Sedghi and N. Shobkolaei, "Common fixed point of maps in complete partial metric spaces," East Asian Mathematical Journal, vol. 29, no. 1, pp. 1-12, 2013.

[18] N. Shobkolaei, S. Sedghi, J. R. Roshan, and I. Altun, "Common fixed point of mappings satisfying almost generalized $(S, T)$ contractive condition in partially ordered partial metric spaces," Applied Mathematics and Computation, vol. 219, no. 2, pp. 443452, 2012.

[19] T. Suzuki, "A generalized Banach contraction principle that characterizes metric completeness," Proceedings of the American Mathematical Society, vol. 136, no. 5, pp. 1861-1869, 2008.

[20] T. Suzuki, "A new type of fixed point theorem in metric spaces," Nonlinear Analysis: Theory, Methods \& Applications, vol. 71, no. 11, pp. 5313-5317, 2009. 


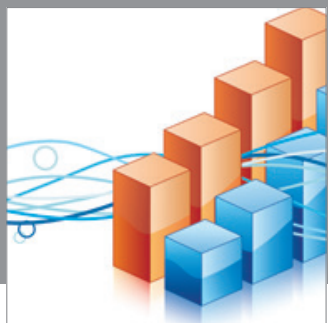

Advances in

Operations Research

mansans

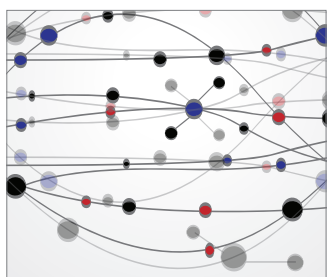

The Scientific World Journal
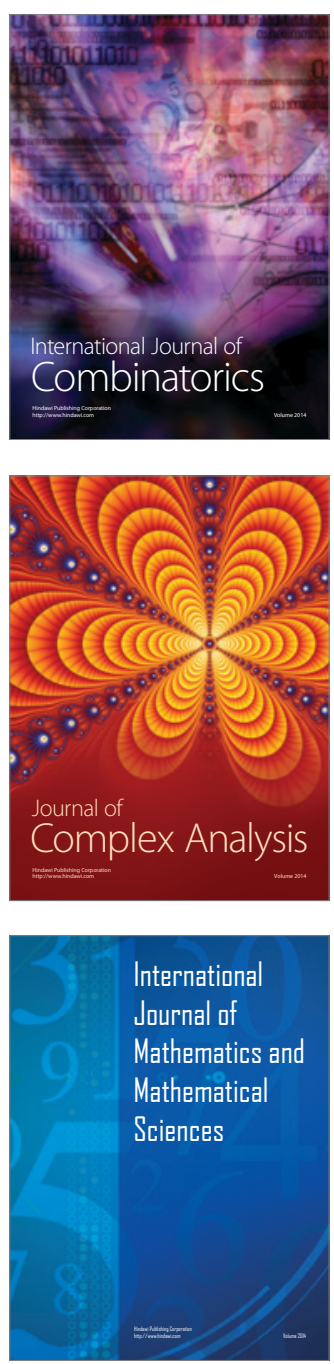
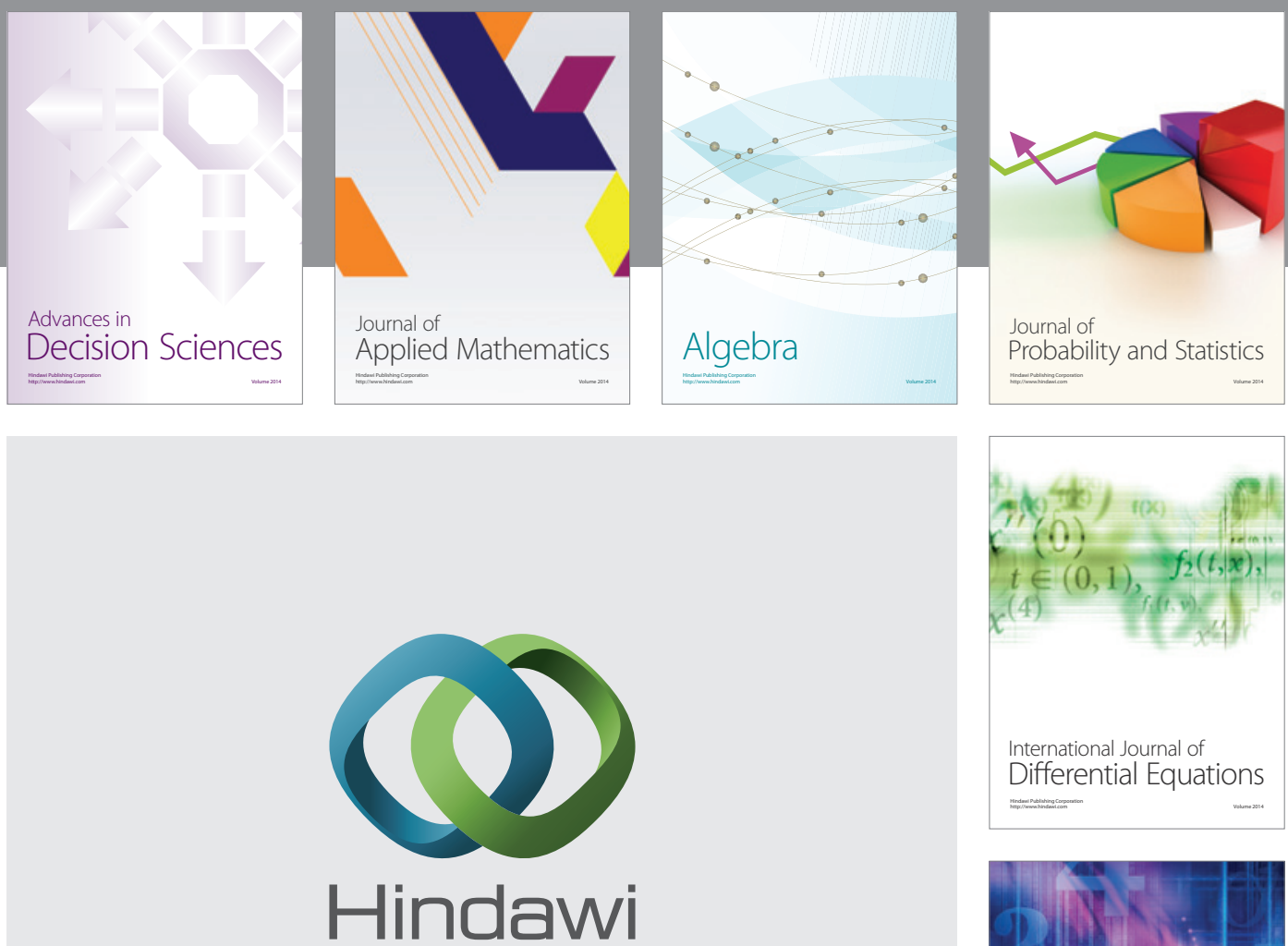

Submit your manuscripts at http://www.hindawi.com
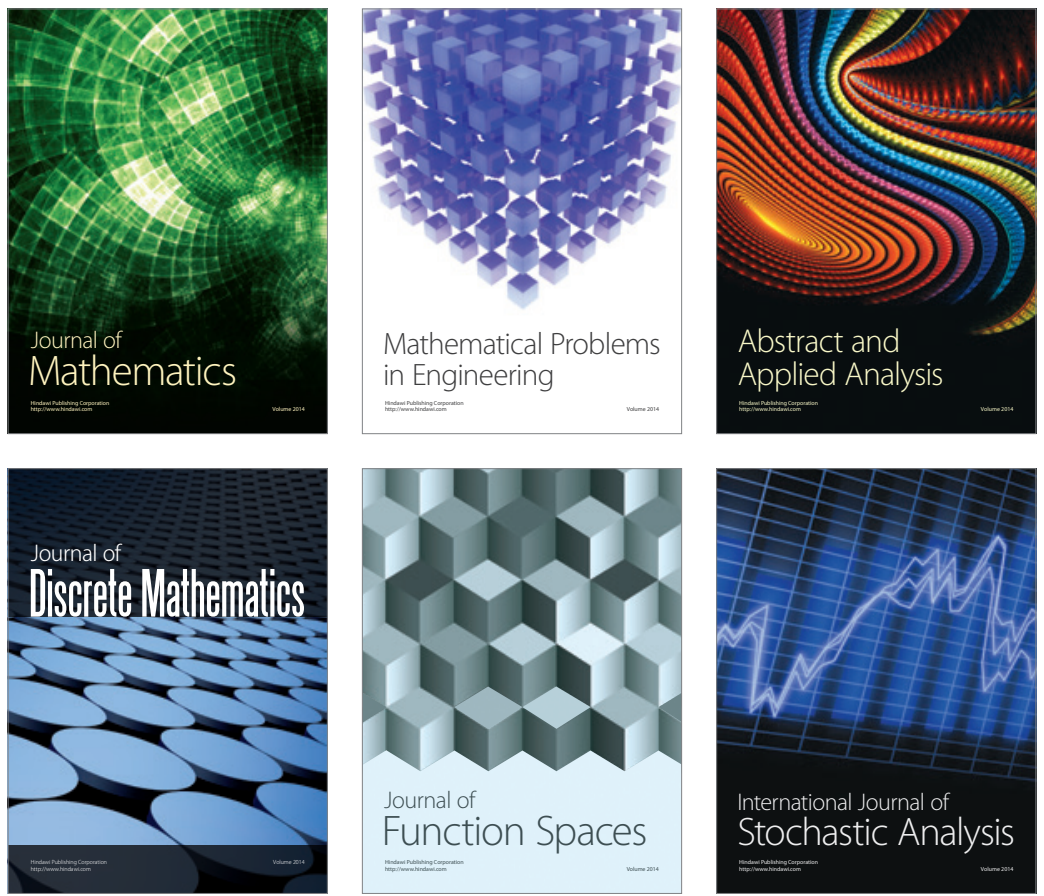

Journal of

Function Spaces

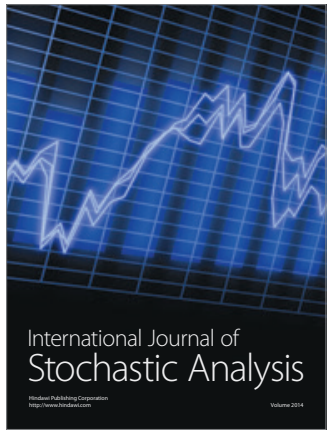

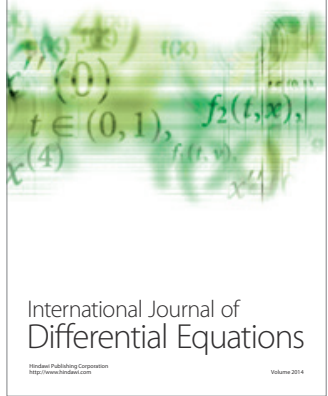
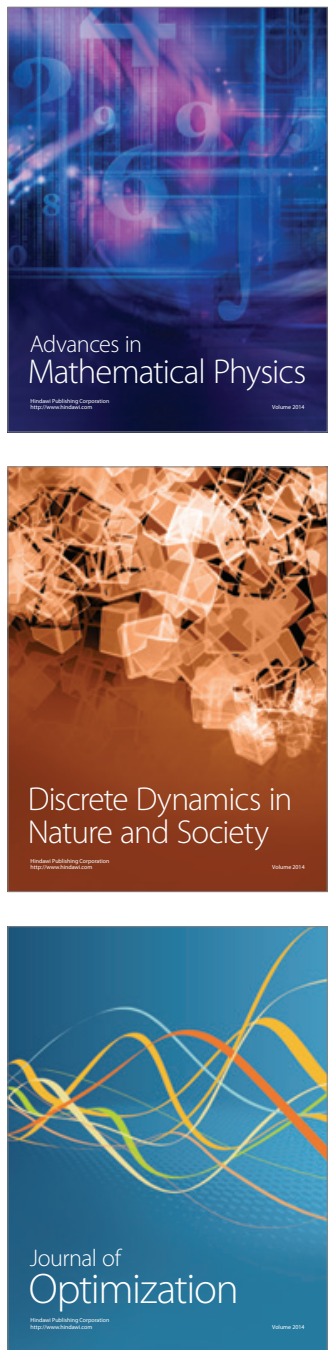